\title{
Electron Transport Through Homopeptides: Are They Really Good Conductors?
}

\author{
Linda A. Zotti*io and Juan Carlos Cuevas*
}

Departamento de Física Teórica de la Materia Condensada and Condensed Matter Physics Center (IFIMAC), Universidad Autónoma de Madrid, Ciudad Universitaria de Cantoblanco, E-28049 Madrid, Spain

\section{Supporting Information}

\begin{abstract}
Motivated by recent experiments, we performed a theoretical study of electron transport through single-molecule junctions incorporating four kinds of homopeptides (based on alanine, glutamic acid, lysine, and tryptophan). Our results suggest that these molecules are rather insulating and operate in off-resonance tunneling as their main transport mechanism. We ascribe their poor performance as conductors to the high localization of their frontier orbitals. We found that binding scenarios in which side chains lie on the side of gold protuberances could give rise to an increase in conductance with respect to end-to-end binding configurations. These findings provide an insight into the conductance mechanism of the building blocks of proteins and identify key issues that need to be further investigated.
\end{abstract}

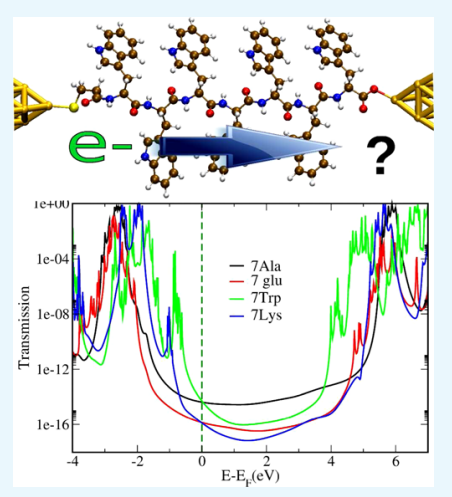

\section{INTRODUCTION}

Over the last decade, increasing interest has been growing on electron transport through biosystems. ${ }^{1-12}$ Until recently, most of the experimental and theoretical studies of the electron flow in biosystems had aimed at understanding the electron transfer taking place in several important biological processes, such as photosynthesis and aerobic respiration. ${ }^{13}$ However, the hope of building novel bio-nanoelectronic devices based on biosystems has prompted the study of these systems as incorporated in solid-state junctions. ${ }^{14}$ The design of such devices would take advantage of the redox and optical functionalities of these systems as well as their chemical recognition and self-assembly properties. In particular, proteins have attracted remarkable attention because of their rich physical (electrical and optical) properties. Their chemistry offers, for instance, endless possibilities in terms of selective binding and self-assembly. Moreover, it makes it possible to tune their properties by means of biochemical modifications. ${ }^{15}$ Electron transport through protein-based junctions was found to be surprisingly efficient, ${ }^{16}$ showing a conductance comparable to that of much shorter alkanedithiol chains (for a detailed review, see ref 1 ). In addition, the study of proteins in solid-state devices could shed new light on the charge-transfer mechanisms of proteins in biological settings despite differences in the environments. Nevertheless, the exact mechanism of transport through proteins remains by and large unknown and is still under debate. ${ }^{1,17}$ To this aim, the study of electron transport through the building blocks of proteins, namely, amino acids, peptides (short chains of amino acids), and polypeptides, is crucial. Furthermore, over the years, these systems have revealed interesting properties. ${ }^{13,18-26}$ Theoretical studies on transport through oligopeptides ${ }^{19,27}$ suggested that orbitals associated with peptide bonds can give rise to states that extend across the entire molecular nanowire and that can be brought into resonance with the Fermi level; moreover, current rectification can be achieved due to the intrinsic asymmetry of the peptide chains. In ref 22, it was shown that rates of charge tunneling along self-assembled monolayers of oligoglycine are comparable to those along SAMs of oligophenyl groups of comparable length. Doping a 7-alanine by one tryptophane ${ }^{28}$ was found to enhance transport by an order of magnitude. Individual amino acids were compared, ${ }^{29}$ reporting similar conductances for glycine, alanine, and proline. Numerous other experimental studies focused on identifying the transport mechanism of peptides (tunneling vs hopping) and the role of linkers as well as chemical and structural modification (see ref 13 and the references therein). In particular, in ref 18 , the current through four families of heptapeptides was studied thoroughly by combining theory and experiments. The current measurements were performed on oligo-homopeptide monolayers held between gold electrodes. In particular, heptatryptophan (7Trp) was found to be the best conductor compared to heptaalanine (7Ala), heptaglutamic acid (7Glu), and heptalysine (7Lys), its current being larger than for the others by up to 1 or 2 orders of magnitude. Upon analysis of the gas-phase orbitals of these compounds, such a relationship was ascribed to their different energetic distribution and spatial localization. The current values reported correspond to conductance values that are, for some of the molecules, higher than those of alkanes of similar length. Moreover, for instance, the length-

Received: December 4, 2017

Accepted: March 26, 2018

Published: April 3, 2018 
dependent conductance decay for 7Trp was found to be slightly smaller than those in fully saturated molecules. Such results are quite intriguing and indicate that these systems are valid candidates to be employed in the field of molecular electronics. However, analyses at the level of single molecule and complete transport calculations, which would better elucidate the conductance properties of these molecules, are still missing to date. This prompted us to try shedding further light on these molecules to contribute to the currently ongoing debate on whether peptides can really play an active role in electron transport. For this reason, we performed a theoretical study on the same peptide-based compounds as those studied in ref 18 . In our work, we take, however, full account of the coupling to the metal electrodes by combining density functional theory (DFT) and nonequilibrium Green's function technique. It is worth stressing that this study does not aim at reproducing the exact conditions present in the experiments ${ }^{18}$ and the corresponding results: this is certainly not possible because of the experimental uncertainties related to, for instance, the number of contacted molecules, the uniformity of the selfassembled monolayers, or the interaction between molecules. For these reasons, and to establish a fair comparison across molecules, we shall rather focus on the analysis of singlemolecule junctions based on these homopeptides. The central conclusion of our work is that, in the context of single-molecule junctions, these peptides exhibit rather low conductance compared to other organic molecules of similar length, and this behavior in turn originates from the high localization of their frontier orbitals.

\section{RESULTS AND DISCUSSION}

Peptides (except for cyclic peptides) have an "N-terminal" and a "C-terminal" residue at their end, corresponding to the presence of an amine $\left(\mathrm{NH}_{2}\right)$ and a carboxyl $(\mathrm{COOH})$ group. Each of the four homopeptides studied in this work is composed of seven identical amino acids (Ala, Trp, Glu, and Lys) and carries, at the N-terminus, a sulfur atom through which it binds to gold (such a bond was proven, in the experiments, ${ }^{18}$ by inelastic electron tunneling spectroscopy measurements performed on the monolayer; however, no clear information was obtained about the type of binding established at the C-terminus side after the formation of the junction). To enable comparison of the theoretical conductance values, we first built $\mathrm{Au}$-homopeptide-Au junctions with identical binding geometry for all four heptapeptides, namely, through the $\mathrm{S}$ atom at the $\mathrm{N}$-terminus side and through the $\mathrm{O}$ atom at the C-terminus side, both atoms being connected to an undercoordinated gold atom of pyramidal electrodes. Following previous literature, the $\mathrm{H}$ atom of the $\mathrm{COOH}$ group was removed. ${ }^{30-35}$ The so-built geometries for the four junctions are shown in Figure 1, and the corresponding gap-corrected zero-bias transmission as a function of energy is displayed in Figure 2. In the following, we will focus on coherent transport to study the relationship between charge transfer and the ground-state electronic structure of the molecules.

This means that our calculations particularly apply to lowtemperature experiments, in which thermal fluctuations are very unlikely to play a role. As an aside, we also note that an exponential length dependence observed for the current indicated off-resonance tunneling as the dominant conductance mechanism in the experiments. ${ }^{18}$ In the coherent regime, following the Landauer approach, the conductance is given by $G=G_{0} T\left(E_{\mathrm{F}}\right)$, where $G_{0}=2 e^{2} / h$ is the quantum of conductance
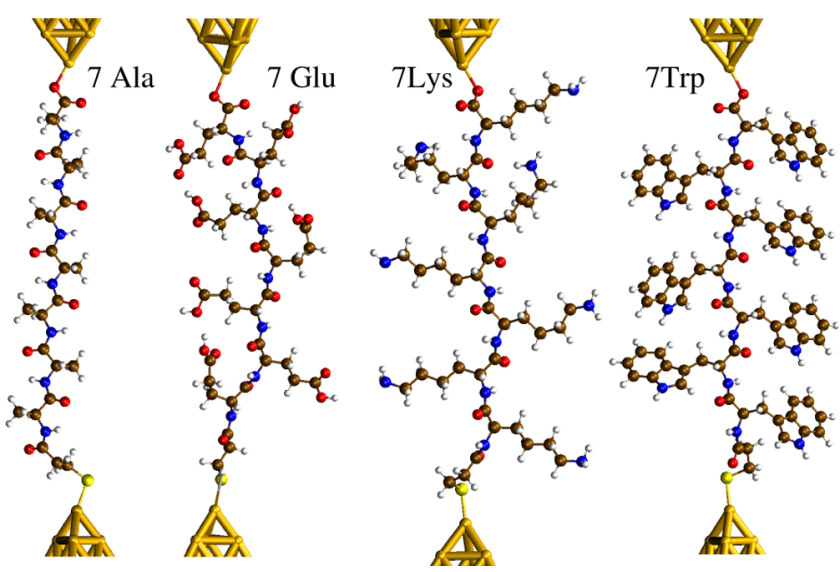

Figure 1. Junctions embedding 7 Ala, 7Glu, 7Lys, and 7Trp, connected to gold electrodes in a top position through an $\mathrm{S}$ atom at one end and an $\mathrm{O}$ atom at the other end.

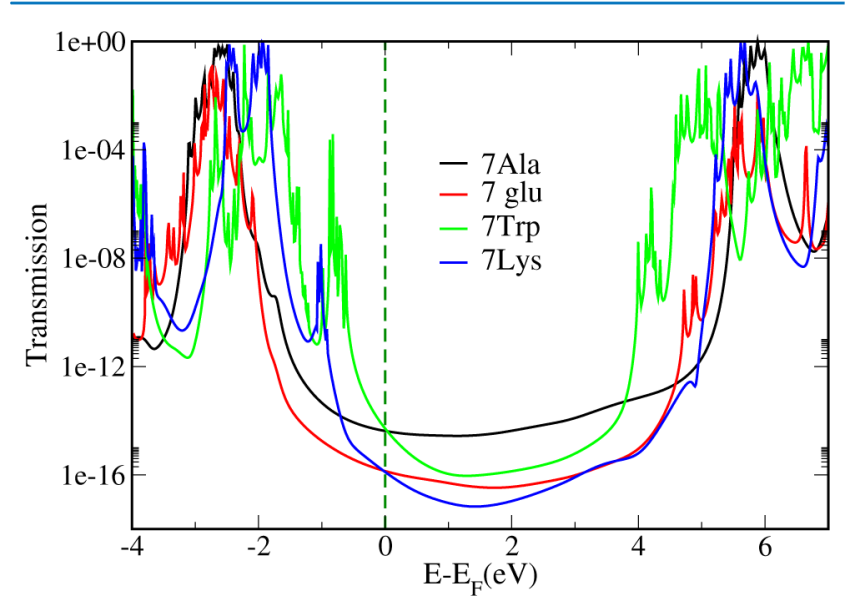

Figure 2. Zero-bias transmission as a function of energy for 7Ala, 7Glu, 7Lys, and 7Trp in the geometries shown in Figure 1 (the corresponding conductance values are, in units of $G_{0}, 4.2 \times 10^{-15}, 1.4$ $\times 10^{-16}, 1.3 \times 10^{-16}$, and $5.4 \times 10^{-15}$; the vertical dashed line indicates the Fermi energy).

and $T\left(E_{\mathrm{F}}\right)$ is the transmission at the Fermi energy (given in turn by the sum over the energy-dependent eigenvalues of the transmission matrix). For all curves in Figure 2, it is possible to observe that the current flows through the highest occupied molecular orbital (HOMO). For 7Trp, 7Lys, and 7Glu, the conductance values (given by the transmission at the Fermi level) follow the trend observed in the experiments $\left(G_{\operatorname{Trp}}>G_{\text {Lys }}\right.$ $\sim G_{\text {Glu }}$ see Table 1). Nevertheless, 7Ala, which the experiments indicate to give the lowest conductance among all, shows, in our simulations, a conductance comparable to that of 7Trp.

Table 1. Experimental Current Values ${ }^{18}$ at $0.1 \mathrm{~V}$ (Error Bars Have Been Neglected); Corresponding Conductance (in Units of $G_{0}$ ); Approximate Total Number of Peptides $N$ in Contact Area (See Ref 18 for More Details); and Conductance per Peptide

$\begin{array}{lcccc} & I(\mathrm{~A}) & G\left(G_{0}\right) & N & G / N \\ 7 \text { Ala } & 4.2 \times 10^{-11} & 5.42 \times 10^{-6} & 5 \times 10^{4} & 1.08 \times 10^{-10} \\ 7 \text { Glu } & 1.9 \times 10^{-10} & 2.45 \times 10^{-5} & 5 \times 10^{3} & 4.90 \times 10^{-9} \\ 7 \text { Lys } & 3.0 \times 10^{-10} & 3.87 \times 10^{-5} & 5 \times 10^{3} & 7.74 \times 10^{-9} \\ 7 \text { Trp } & 9.2 \times 10^{-10} & 1.19 \times 10^{-4} & 5 \times 10^{3} & 2.37 \times 10^{-8}\end{array}$




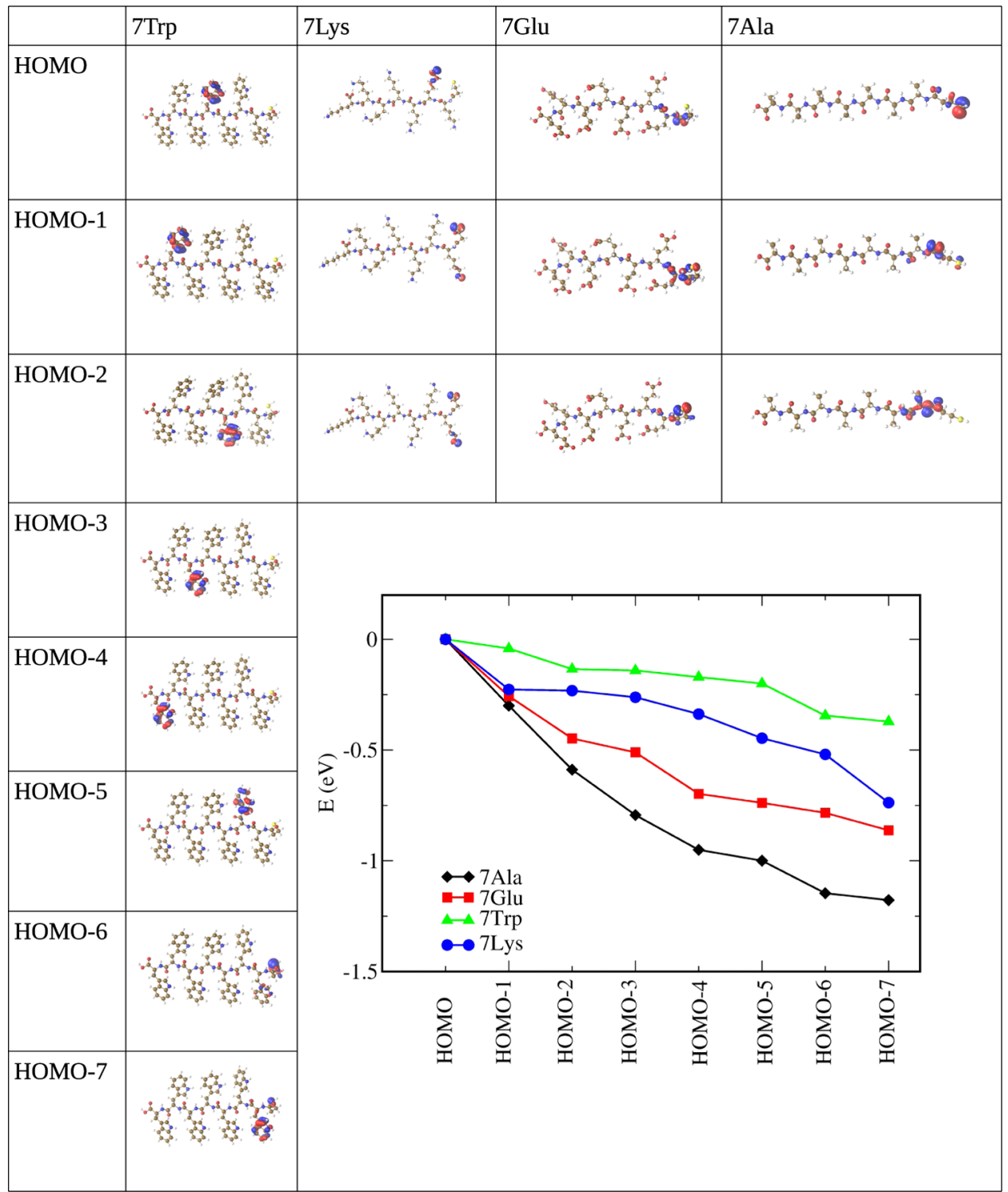

Figure 3. Gas-phase occupied orbitals for 7Ala, 7Glu, 7Lys, and 7Trp and the corresponding energetic positions. For 7Trp, all orbitals down to HOMO-7 are shown since they are energetically competing. All values have been shifted so as to set HOMO to zero for all molecules for a better comparison.

Moreover, the theoretical conductance values (in units of $G_{0}$, $4.2 \times 10^{-15}, 1.4 \times 10^{-16}, 1.3 \times 10^{-16}$, and $5.4 \times 10^{-15}$ for 7 Ala, 7Glu, 7Lys, and 7Trp, respectively) appear to be much lower than those observed experimentally by more than 5 orders of magnitude; the experimental values are summarized in Table 1. The computed values are clearly much lower than those measured for conjugated molecules of similar length $\left(\sim 10^{-7} G_{0}\right)^{36}$ and also even lower than the conductance values obtained (by extrapolation) for alkanes of comparable length $\left(\sim 10^{-10} G_{0}\right)^{37}$

Our calculations are based on single-molecule junctions and thus do not take into account dipole-dipole interactions that could be present between peptides inside a monolayer and that might lower the HOMO-lowest unoccupied molecular orbital (LUMO) gap by shifting the occupied (unoccupied) levels to higher (lower) energies. ${ }^{38}$ This would yield an increase in conductance, which cannot be evaluated in our model and neither can we estimate whether such effect would compensate the quantitative disagreement that we observe between our results and the experiments. Nevertheless, this goes beyond the scope of the present work, which aims at identifying the conductance properties of these molecules as isolated. More precisely, our simulations aim at predicting the results in singlemolecule experiments, where establishing a fairer comparison across peptides would be possible. They thus do not address issues that might be important in SAM-based junctions, such as the interaction between molecules, the inhomogeneities in the junctions, or the potential modification of the metal work function due to the interaction with the molecular monolayer. ${ }^{39}$ We note also that the structure of these heptapeptides is very flexible; thus, a complete analysis should take into account a broader range of structures (possibly extracted by molecular dynamics calculations). In fact, although our simulations are meant to reproduce experiments at low temperature, quenching of the molecular ensemble at low temperature might induce the different molecules to freeze in different conformations; this, together with the frequency of their occurrence, could be sampled by molecular dynamics simulations and give rise to a 
broader conductance range than that shown by our simulations. For further insight, we now turn to analyze the orbitals of the four heptapeptides in the gas phase (Figure 3). It can be observed that the orbitals corresponding to the highest occupied levels, through which the current flows, are all localized on regions that have a much smaller spatial extension than the overall length (of around $25 \pm 2 \AA$ ). This is obviously reflected in the low conductance values obtained by our transport calculations. Figure 3 also shows the Kohn-Sham energy values of the seven highest occupied gas-phase orbitals (from HOMO to HOMO-7). In the case of 7Trp, it can be observed that they line up at very similar energies, each orbital being localized on a different tryptophan unit. The same does not apply to the other three molecules, for which the energy is seen to decrease at a higher rate across the orbitals. For 7Trp, the similar energy alignment of these orbitals gives rise to many sharp transmission resonances that are very close to each other (Figure 2). Indeed, it was suggested ${ }^{18}$ that these orbitals would form a single HOMO band, with an overall charge density delocalized over the entire molecule, which could explain the efficient tunneling process observed experimentally for 7Trp. However, our results suggest that, although the corresponding transmission resonances are indeed energetically very close to each other, the overall conductance remains low. It remains to be seen if different combinations of exchange-correlation functionals and basis sets would change these conclusions by increasing the level of delocalization of each individual orbital, thus giving rise to the formation of a band and therefore increasing the overall conductance of 7Trp. We also note that the asymmetry at the two ends for 7Lys, 7Glu, and 7Ala seems to induce the charge to be localized on one side of the molecule, as we can observe in Figure 3 for these three molecules. Such an effect is clearly modified in the case of 7Trp, where the side chains enable different charge localizations. This increases the overall delocalization of the band, which would result by a combination of the seven highest molecular orbitals. Thermal fluctuations have not been considered in our simulations. However, measurements performed at 7 and 300 $\mathrm{K}$ did not reveal any particular dependence on temperature (Figure $3 c$ in ref 18). Our zero-bias calculations do not take into account the effect of applying a voltage. Nevertheless, this is not expected to change our main conclusions since the voltage corresponding to the experimental values reported in Table $1(0.1 \mathrm{~V})$ corresponds to the linear regime; indeed, the transmission resonances of the curves shown in Figure 2 are well beyond the energy window that would correspond to such a bias. In particular, the transmission curves are relatively constant around the Fermi energy in an energy range larger than $0.1 \mathrm{eV}$, which clearly justifies our approximation.

It is also worth stressing that the $S$ atom used to bind these heptapeptides to gold is not originally present in natural peptides, and recent studies have been devoted to understanding the conductive properties of peptides in the absence of additional anchoring groups and in their native solvent (water). ${ }^{40}$ Thiol is one of the most widely used end groups in molecular electronics (see ref 41 and references therein) as it ensures robust binding to the electrodes. However, it is known that anchoring groups can affect the conductance by shifting the frontier molecular orbitals and by modifying the coupling to the leads. ${ }^{42}$ For instance, use of $S$ atoms has been shown to lead to different conductances depending on whether binding through $\mathrm{S}$ or $\mathrm{SH}$ is established. ${ }^{43}$ However, it is very unlikely that a different binding group would turn these molecules into much better conductors, as low conductance has also been detected in unmodified peptides. ${ }^{40}$

For the tryptophan-based structure, we also studied the length dependence of the conductance by changing the number of tryptophan units (from 1 to 7 ). In ref 18 , an exponential dependence was observed, with a $\beta$ decay constant of $0.58 \AA^{-1}$, which was found to be independent of temperature, suggesting tunneling as the dominant transport mechanism. The theoretical transmission curves are shown in Figure 4. The

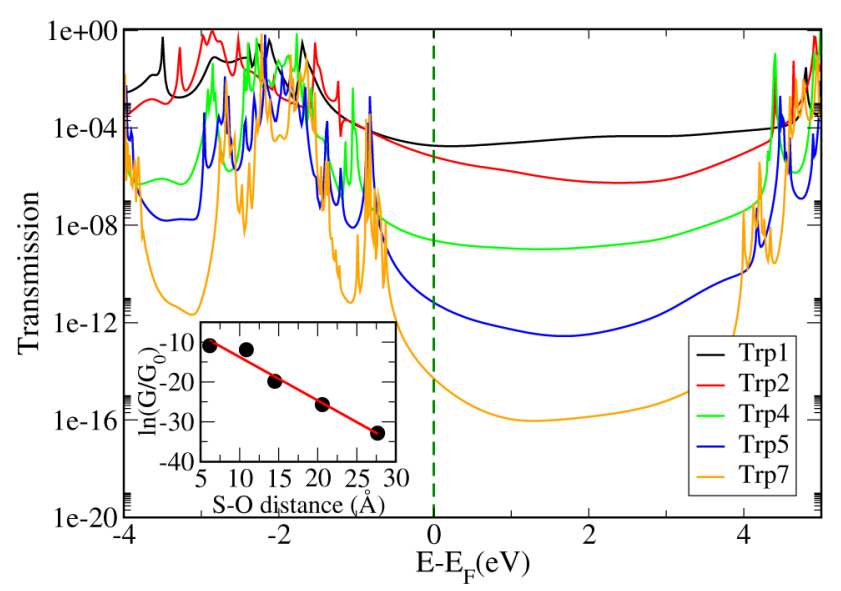

Figure 4. Transmission as a function of energy for homopeptide consisting of 1-7 Trp units. In the inset, conductance as a function of the distance between the terminal $\mathrm{S}$ and the $\mathrm{O}$ atom connected to the gold is reported, together with the corresponding linear fit (red solid line).

corresponding $\beta$ value $\left(1.1 \AA^{-1}\right)$ is higher than the experimentally obtained value and the values reported in the literature for fully saturated molecules $\left(0.8-1 \AA^{-1}\right)^{44}$ and conjugated ones $\left(0.2-0.6 \AA^{-1}\right) .{ }^{45,46}$ We will return to the issue of these discrepancies further ahead in the manuscript.

Finally, we want to investigate the conductance behavior of the helical structure of 20Ala, which was also analyzed in ref 18 , with the aim of studying the role of the secondary structure. Due to the importance of helical structures in electron-transfer processes, this topic has been the object of several studies. $^{21,47,48}$ In Figure 5 b, we show the structure of helical 20Ala embedded in the junction, whereas, in Figure 5a, its transmission curve is compared to that of the extended structure of $7 \mathrm{Ala}$, which has similar length. In ref 18 , an increase in conductance of helical 20Ala compared to extended 7Ala was reported. We observed a huge increase (by 10 orders of magnitude, see Supporting Information) in the conductance of helical 20Ala with respect to its extended structure; this is not surprising because the helical shape favors electron transport by increasing the overlap between orbitals. However, we did not observe any increase with respect to $7 \mathrm{Ala}$. The reason for the low conductance can probably be traced back, once again, to the high localization of the relevant orbitals: Figure 5c shows that the six highest occupied molecular orbitals of the gas-phase helical 20Ala are all localized on the side of the C-terminus. This yields a strong asymmetry of the junction, resulting in the corresponding transmission resonances being low. Our model does not take into account the possibility of lifting the spin degeneracy due to the chiral-induced spin selectivity effect, which has previously been observed in helical peptides. ${ }^{48}$ Nevertheless, such an effect would reduce the total 

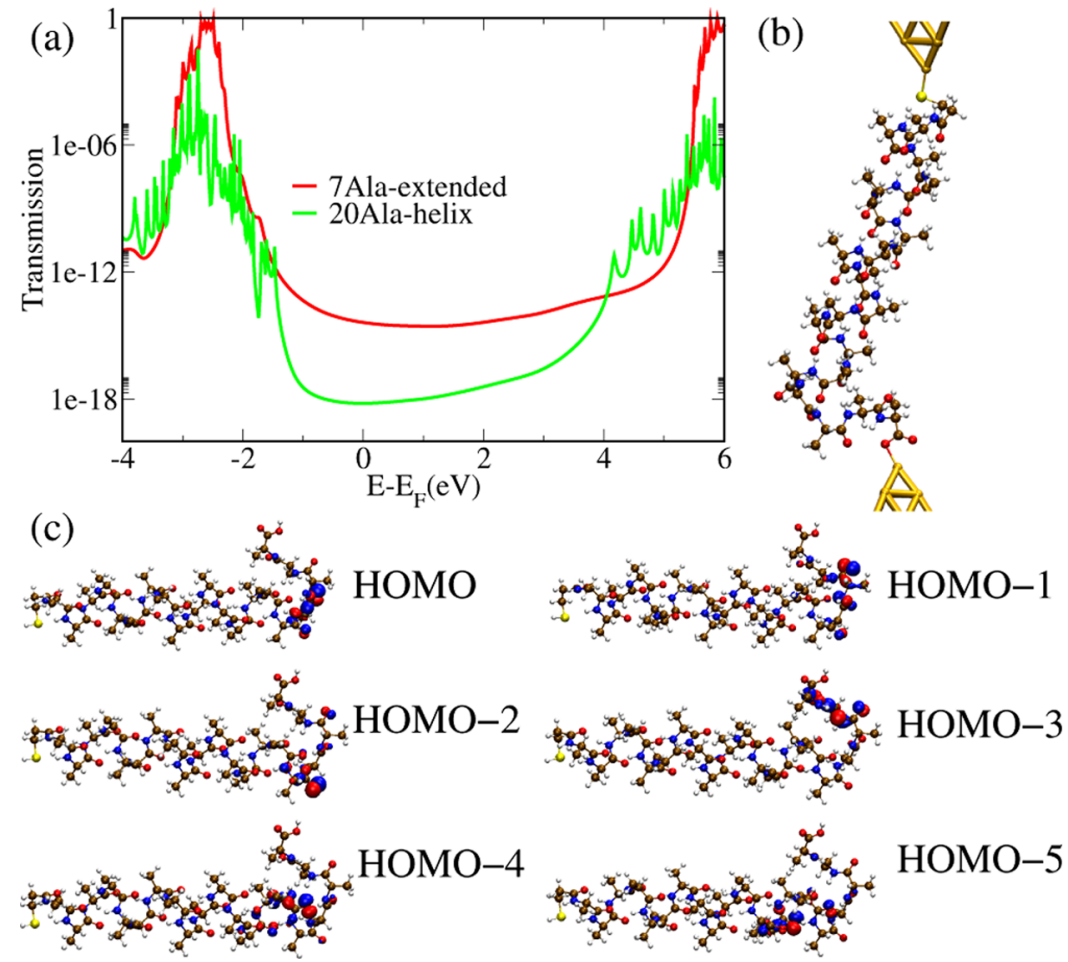

Figure 5. (a) Transmission as a function of energy for extended 7Ala and helical 20Ala; (b) geometrical structure for helical 20Ala in the junction; and (c) six highest occupied gas-phase molecular orbitals for helical 20Ala.

conductance of 20Ala even further, contrary to what was found in the experiments.

To find alternative explanations for the quantitative disagreement observed between theory and experiments (besides the absence of intermolecular interaction effects that are not described here), we investigated the possibility of occurrence of geometries different from those shown in Figure 1, which could lead to higher conductance. In Figure 6, we show other possible binding structures that could be formed. Figure $6 \mathrm{a}, \mathrm{b}$ show geometries in which the gold apex on the C-terminus is close to not only the $\mathrm{S}$ atom but also one of the terminal oxygen atoms; Figure $6 c-f$, instead, show geometries in which the molecular end part on the side of the C-terminus lies parallel to one face of the gold pyramid. Similar structures have been suggested in the past. ${ }^{49,50}$ In the case of 7Lys (Figure 6e), we noted the formation of additional bonds between the nitrogen atoms of the side chains and the gold atoms of the gold pyramid. The corresponding transmission curves are shown in Figure 7 and compared to those obtained for the geometries of Figure 1. We note that, for the transmission curves displayed in Figure 7, no gap correction was applied.

We can observe that geometries where the heptapeptides lie parallel to the face of the gold pyramid generally correspond to an increase in conductance. This is mainly due to a shorter distance between the two gold electrodes (see the Supporting Information for further details). Therefore, we suggest that part of the quantitative disagreement we observed with the experiments for the results reported above could be due to the fact that, when the whole monolayer junction is formed, not all individual single-molecule junctions are formed in such a way that only the extreme parts are bound to gold; instead, some probably end up in configurations similar to those shown in Figure 6, which present higher binding energies than in the end-to-end configurations (see Table S2 in the Supporting

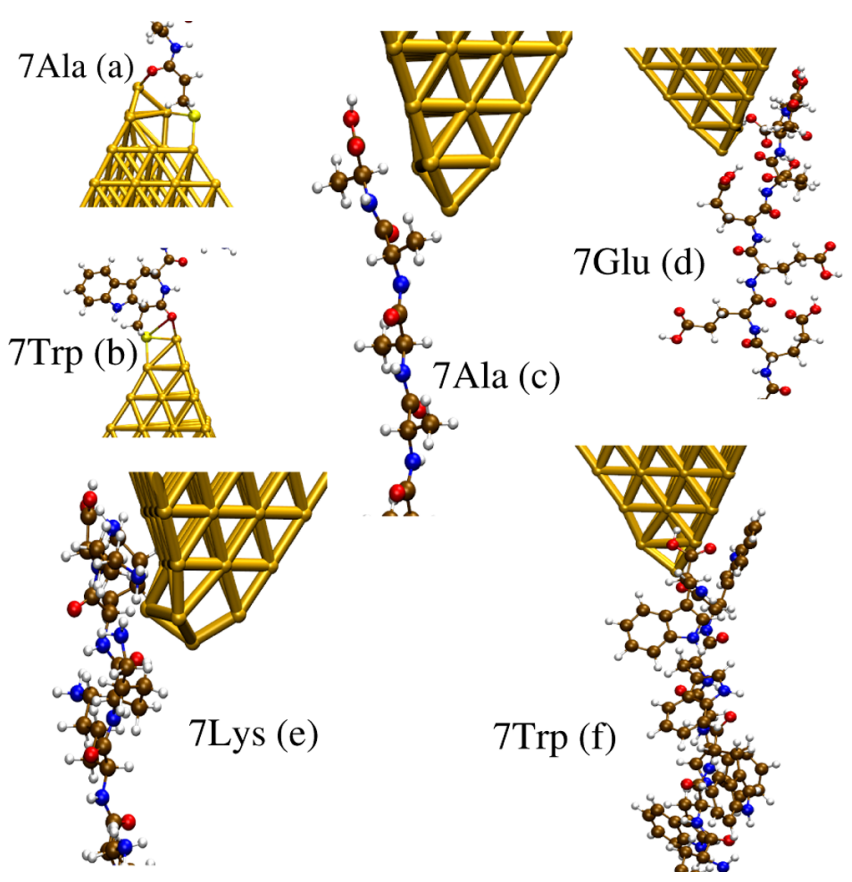

Figure 6. Geometries studied for molecular junctions incorporating 7Ala (a, c), 7Trp (b, f), 7Glu (d), and 7Lys (e), in addition to those shown in Figure 1.

Information). This is plausible because once the top contact is formed upon landing of the gold nanorod on the monolayer, some gold can penetrate between one heptapeptide and the other. The geometries shown in Figure 6 are only an example of the many configurations that could occur and other possible binding scenarios that have here not been considered are possible. ${ }^{51}$ Moreover, the structures of these molecules are 


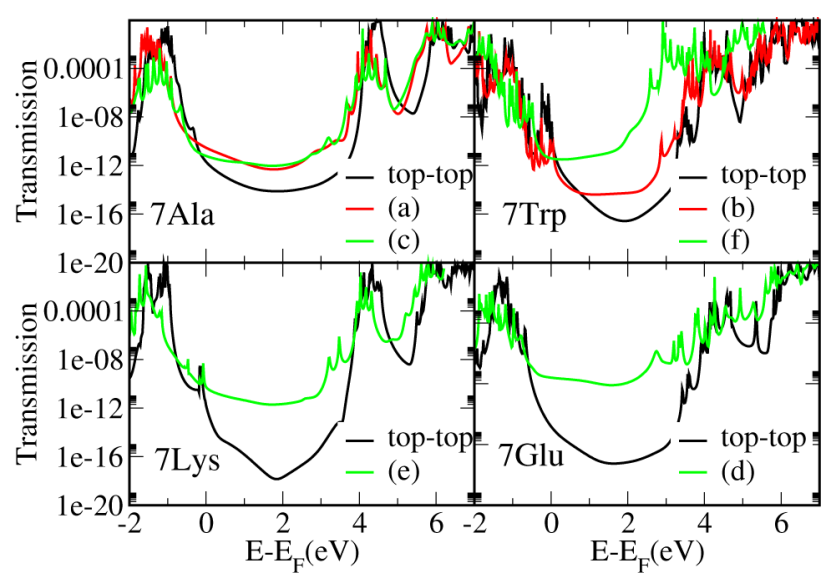

Figure 7. Transmission as a function of energy for the geometries shown in Figure 6a-f. Those labeled as "top-top" correspond to the geometries shown in Figure 1. No HOMO-LUMO gap correction was applied to the transmission curves shown in this figure.

extremely flexible, especially concerning the orientation of the side chains, and can easily experience deformations that can lead them to several kinds of structures. Moreover, for each geometry shown in Figure 6c-e, changing the distance between the gold electrodes would yield a broad range of conductance values. Overall, the complexity of this scenario leads to highlighting the fact that performing single-molecule measurements is highly needed in this case, to single out the contribution from the individual homopeptides and rule out any additional effect due to the interaction with the surrounding molecules.

\section{CONCLUSIONS}

The electron transport through four types of heptapeptides (Ala, Trp, Glu, and Lys) and through shorter analogues of the Trp-based molecule was studied theoretically. We found that these molecules present rather low conductance and we ascribed this to the high localization of their frontier orbitals. Furthermore, we compared our results to those obtained experimentally and reported in previous literature. On the basis of such a comparison, we suggest that a broad range of binding geometries could be established in the junction, thanks to the extreme structural flexibility of these molecules. More importantly, we point out the need for single-molecule measurements on these systems to clarify their transport properties.

\section{THEORETICAL METHODS}

We built molecular junctions consisting of single homopeptides held between gold electrodes. Ab initio calculations based on density functional theory (DFT) were then performed to determine the geometry and the electronic structure of these systems as well as their transport properties. The DFT calculations were carried out using the quantum chemistry code TURBOMOLE, ${ }^{52}$ employing a split-valence basis set with polarization functions for all nonhydrogen atoms ${ }^{53}$ and the BP86 exchange-correlation functional. ${ }^{54}$ We first carried out geometry optimizations of all molecules in the gas phase and subsequently built the gold-molecule-gold junctions by placing the relaxed structures between two $\mathrm{Au}_{20}$ clusters; these junction geometries were then optimized by allowing geometrical relaxation of the molecule and of the four $\mathrm{Au}$ atoms closer to it on each side of the junction. Afterward, further gold layers were added to the optimized structures to extend the size of both clusters to around 60 atoms and to describe correctly the metal-molecule charge transfer as well as the corresponding level alignment. After running single-point calculations on these junctions, we computed the zero-bias conductance of the junctions with the Landauer formalism using Green's function techniques. Within our model, the electrodes are described as semi-infinite surfaces and the Fermi level is obtained by a separate calculation of the electronic structure of the infinite metallic leads. ${ }^{55}$ The HOMO-LUMO gap was corrected by the DFT $+\sum$ method. $^{56,57}$ The DFT-D2 correction for the dispersive forces ${ }^{58}$ was included in the optimization of the geometries shown in Figure 6.

\section{ASSOCIATED CONTENT}

\section{S Supporting Information}

The Supporting Information is available free of charge on the ACS Publications website at DOI: 10.1021/acsomega.7b01917.

Additional information on transmission curves, transmission coefficients, relevant orbitals for the conductance, HOMO-LUMO gap correction, and binding energies (PDF)

\section{AUTHOR INFORMATION}

\section{Corresponding Authors}

*E-mail: linda.zotti@uam.es (L.A.Z.).

*E-mail: juancarlos.cuevas@uam.es (J.C.C.).

ORCID ${ }^{\circ}$

Linda A. Zotti: 0000-0002-5292-6759

Notes

The authors declare no competing financial interest.

\section{ACKNOWLEDGMENTS}

The authors thank Rubén Pérez, Guilherme Vilhena, and Fabian Pauly for fruitful discussions, and Leeor Kronik and Sivan Refaely-Abramson for providing the initial geometrical structures. L.A.Z. was funded by the Spanish MINECO through the grant MAT2014-58982-JIN. J.C.C. acknowledges financial support from the Spanish MINECO (FIS2014-53488-P and FIS2017-84057-P) as well as DFG and SFB767 for sponsoring his stay at the University of Konstanz as Mercator Fellow.

\section{REFERENCES}

(1) Amdursky, N.; Marchak, D.; Sepunaru, L.; Pecht, I.; Sheves, M.; Cahen, D. Electronic transport via proteins. Adv. Mater. 2014, 26, $7142-7161$.

(2) Alfinito, E.; Reggiani, L.; Pousset, J. Proteotronics: Electronic Devices Based on Proteins. Sensors 2015, 3-7.

(3) Chi, Q.; Farver, O.; Ulstrup, J. Long-range protein electron transfer observed at the single-molecule level: In situ mapping of redox-gated tunneling resonance. Proc. Natl. Acad. Sci. U.S.A. 2005, 102, 16203-16208.

(4) Alessandrini, A.; Facci, P. Electron transfer in nanobiodevices. Eur. Polym. J. 2016, 83, 450-466.

(5) Blumberger, J. Recent advances in the theory and molecular simulation of biological electron transfer reactions. Chem. Rev. 2015, 115, 11191-11238.

(6) Baldacchini, C.; Bizzarri, A. R.; Cannistraro, S. Electron transfer, conduction and biorecognition properties of the redox metalloprotein Azurin assembled onto inorganic substrates. Eur. Polym. J. 2016, 83, 407-427. 
(7) Livshits, G. I.; Stern, A.; Rotem, D.; Borovok, N.; Eidelshtein, G.; Migliore, A.; Penzo, E.; Wind, S. J.; Di Felice, R.; Skourtis, S. S.; et al. Long-range charge transport in single G-quadruplex DNA molecules. Nat. Nanotechnol. 2014, 9, 1040-1046.

(8) Xiang, L.; Palma, J. L.; Bruot, C.; Mujica, V.; Ratner, M. A.; Tao, $\mathrm{N}$. Intermediate tunnelling-hopping regime in DNA charge transport. Nat. Chem. 2015, 7, 221-226.

(9) Macchia, E.; Alberga, D.; Manoli, K.; Mangiatordi, G. F.; Magliulo, M.; Palazzo, G.; Giordano, F.; Lattanzi, G.; Torsi, L. Organic bioelectronics probing conformational changes in surface confined proteins. Sci. Rep. 2016, 6, No. 28085.

(10) Bâldea, I. Important insight into electron transfer in singlemolecule junctions based on redox metalloproteins from transition voltage spectroscopy. J. Phys. Chem. C 2013, 117, 25798-25804.

(11) Yamana, K.; Erbe, A.; Barton, J. K.; Furst, A. L.; Grodick, M. A.; Choi, J.; Majima, T. DNA Wires and Electron Transport Through DNA; John Wiley \& Sons, Ltd, 2015; pp 79-136.

(12) Xiao, Y.; Zhang, E.; Zhang, J.; Dai, Y.; Yang, Z.; Christensen, H. E.; Ulstrup, J.; Zhao, F. Extracellular polymeric substances are transient media for microbial extracellular electron transfer. Sci. Adv. 2017, 3, No. e1700623.

(13) Shah, A.; Adhikari, B.; Martic, S.; Munir, A.; Shahzad, S.; Ahmad, K.; Kraatz, H.-B. Electron transfer in peptides. Chem. Soc. Rev. 2015, 44, 1015-1027.

(14) Maruccio, G. Molecular electronics: Protein transistors strike gold. Nat. Nanotechnol. 2012, 7, 147-148.

(15) Cuevas, J. C.; Scheer, E. Molecular Clectronics: An Introduction to Theory and Experiment, 2nd ed.; World Scientific, 2017; Vol. 15.

(16) Artés, J. M.; Díez-Pérez, I.; Gorostiza, P. Transistor-like behavior of single metalloprotein junctions. Nano Lett. 2012, 12, 2679-2684.

(17) Ruiz, M. P.; Aragones, A. C.; Camarero, N.; Vilhena, J.; Ortega, M.; Zotti, L. A.; Perez, R.; Cuevas, J. C.; Gorostiza, P.; Díez-Pérez, I. Bioengineering a Single-Protein Junction. J. Am. Chem. Soc. 2017, 139, $15337-15346$.

(18) Sepunaru, L.; Refaely-Abramson, S.; Lovrincic, R.; Gavrilov, Y.; Agrawal, P.; Levy, Y.; Kronik, L.; Pecht, I.; Sheves, M.; Cahen, D. Electronic transport via homopeptides: The role of side chains and secondary structure. J. Am. Chem. Soc. 2015, 137, 9617-9626.

(19) Cardamone, D. M.; Kirczenow, G. Single-molecule device prototypes for protein-based nanoelectronics: Negative differential resistance and current rectification in oligopeptides. Phys. Rev. B 2008, 77, No. 165403.

(20) Li, W.-Q.; Huang, B.; Huang, M.-L.; Peng, L.-L.; Hong, Z.-W.; Zheng, J.-F.; Chen, W.-B.; Li, J.-F.; Zhou, X.-S. Detecting Electron Transport of Amino Acids by Using Conductance Measurement. Sensors 2017, 17, No. 811.

(21) Amdursky, N. Electron Transfer across Helical Peptides. ChemPlusChem 2015, 80, 1075-1095.

(22) Baghbanzadeh, M.; Bowers, C. M.; Rappoport, D.; Żaba, T.; Gonidec, M.; Al-Sayah, M. H.; Cyganik, P.; Aspuru-Guzik, A.; Whitesides, G. M. Charge tunneling along short oligoglycine chains. Angew. Chem., Int. Ed. 2015, 54, 14743-14747.

(23) Berstis, L.; Beckham, G. T.; Crowley, M. F. Electronic coupling through natural amino acids. J. Chem. Phys. 2015, 143, No. 225102.

(24) Yu, J.; Horsley, J. R.; Abell, A. D. Turning electron transfer 'onoff in peptides through side-bridge gating. Electrochim. Acta 2016, 209, $65-74$.

(25) Ivnitski, D.; Amit, M.; Silberbush, O.; Atsmon-Raz, Y.; Nanda, J.; Cohen-Luria, R.; Miller, Y.; Ashkenasy, G.; Ashkenasy, N. The Strong Influence of Structure Polymorphism on the Conductivity of Peptide Fibrils. Angew. Chem. 2016, 128, 10142-10146.

(26) Gao, J.; Müller, P.; Wang, M.; Eckhardt, S.; Lauz, M.; Fromm, K. M.; Giese, B. Electron transfer in peptides: the influence of charged amino acids. Angew. Chem., Int. Ed. 2011, 50, 1926-1930.

(27) Cardamone, D. M.; Kirczenow, G. Electrochemically gated oligopeptide nanowires bridging gold electrodes: novel bio-nanoelectronic switches operating in aqueous electrolytic environments. Nano Lett. 2010, 10, 1158-1162.
(28) Guo, C.; Yu, X.; Refaely-Abramson, S.; Sepunaru, L.; Bendikov, T.; Pecht, I.; Kronik, L.; Vilan, A.; Sheves, M.; Cahen, D. Tuning electronic transport via hepta-alanine peptides junction by tryptophan doping. Proc. Natl. Acad. Sci. U.S.A. 2016, 113, 10785-10790.

(29) Juhaniewicz, J.; Sek, S. Peptide molecular junctions: Electron transmission through individual amino acid residues. J. Electroanal. Chem. 2010, 649, 83-88.

(30) Martín, S.; Haiss, W.; Higgins, S.; Cea, P.; Lopez, M. C.; Nichols, R. J. A comprehensive study of the single molecule conductance of $\alpha, \omega$-dicarboxylic acid-terminated alkanes. J. Phys. Chem. C 2008, 112, 3941-3948.

(31) Paik, W.-k.; Han, S.; Shin, W.; Kim, Y. Adsorption of carboxylic acids on gold by anodic reaction. Langmuir 2003, 19, 4211-4216.

(32) Provorse, M. R.; Aikens, C. M. Binding of carboxylates to gold nanoparticles: a theoretical study of the adsorption of formate on $\mathrm{Au}$ 20. Comput. Theor. Chem. 2012, 987, 16-21.

(33) Han, S. W.; Joo, S. W.; Ha, T. H.; Kim, Y.; Kim, K. Adsorption characteristics of anthraquinone-2-carboxylic acid on gold. J. Phys. Chem. B 2000, 104, 11987-11995.

(34) Bao, D.-L.; Liu, R.; Leng, J.-C.; Zuo, X.; Jiao, Y.; Li, Z.-L.; Wang, C.-K. Theoretical study on mechanical and electron-transport properties of conjugated molecular junctions with carboxylic or methyl sulfide links. Phys. Lett. A 2014, 378, 1290-1295.

(35) Ahn, S.; Aradhya, S. V.; Klausen, R. S.; Capozzi, B.; Roy, X.; Steigerwald, M. L.; Nuckolls, C.; Venkataraman, L. Electronic transport and mechanical stability of carboxyl linked single-molecule junctions. Phys. Chem. Chem. Phys. 2012, 14, 13841-13845.

(36) Zhao, X.; Huang, C.; Gulcur, M.; Batsanov, A. S.; Baghernejad, M.; Hong, W.; Bryce, M. R.; Wandlowski, T. Oligo (aryleneethynylene) s with terminal pyridyl groups: synthesis and length dependence of the tunneling-to-hopping transition of single-molecule conductances. Chem. Mater. 2013, 25, 4340-4347.

(37) Leary, E.; van Zalinge, H.; Higgins, S. J.; Nichols, R. J.; de Biani, F. F.; Leoni, P.; Marchetti, L.; Zanello, P. A molecular wire incorporating a robust hexanuclear platinum cluster. Phys. Chem. Chem. Phys. 2009, 11, 5198-5202.

(38) Rissner, F.; Natan, A.; Egger, D. A.; Hofmann, O. T.; Kronik, L.; Zojer, E. Dimensionality effects in the electronic structure of organic semiconductors consisting of polar repeat units. Org. Electron. 2012, 13, 3165-3176.

(39) Parker, C. R.; Leary, E.; Frisenda, R.; Wei, Z.; Jennum, K. S.; Glibstrup, E.; Abrahamsen, P. B.; Santella, M.; Christensen, M. A.; Della Pia, E. A.; et al. A Comprehensive Study of Extended Tetrathiafulvalene Cruciform Molecules for Molecular Electronics: Synthesis and Electrical Transport Measurements. J. Am. Chem. Soc. 2014, 136, 16497-16507.

(40) Brisendine, J. M.; Refaely-Abramson, S.; Liu, Z.-F.; Cui, J.; Ng, F.; Neaton, J. B.; Koder, R. L.; Venkataraman, L. Probing Charge Transport through Peptide Bonds. J. Phys. Chem. Lett. 2018, 9, 763767.

(41) Leary, E.; Zotti, L. A.; Miguel, D.; Márquez, I. R.; PalominoRuiz, L.; Cuerva, J. M.; Rubio-Bollinger, G.; González, M. T.; Agrait, $\mathrm{N}$. The Role of Oligomeric Gold-Thiolate Units in Single-Molecule Junctions of Thiol-Anchored Molecules. J. Phys. Chem. C 2018, 122, 3211-3218.

(42) Zotti, L. A.; Kirchner, T.; Cuevas, J.; Pauly, F.; Huhn, T.; Scheer, E.; Erbe, A. Revealing the Role of Anchoring Groups in the Electrical Conduction Through Single Molecule Junctions. Small 2010, 6, 1529-1535.

(43) Souza, A. d. M.; Rungger, I.; Pontes, R. B.; Rocha, A. R.; da Silva, A. J. R.; Schwingenschloegl, U.; Sanvito, S. Stretching of BDTgold molecular junctions: thiol or thiolate termination? Nanoscale 2014, 6, 14495-14507.

(44) Yaffe, O.; Qi, Y.; Scheres, L.; Puniredd, S. R.; Segev, L.; Ely, T.; Haick, H.; Zuilhof, H.; Vilan, A.; Kronik, L.; et al. Charge transport across metal/molecular (alkyl) monolayer-Si junctions is dominated by the LUMO level. Phys. Rev. B 2012, 85, No. 045433. 
(45) Salomon, A.; Cahen, D.; Lindsay, S.; Tomfohr, J.; Engelkes, V.; Frisbie, C. Comparison of Electronic Transport Measurements on Organic Molecules. Adv. Mater. 2003, 15, 1881-1890.

(46) Holmlin, R. E.; Haag, R.; Chabinyc, M. L.; Ismagilov, R. F.; Cohen, A. E.; Terfort, A.; Rampi, M. A.; Whitesides, G. M. Electron Transport through Thin Organic Films in Metal-Insulator-Metal Junctions Based on Self-Assembled Monolayers. J. Am. Chem. Soc. 2001, 123, 5075-5085.

(47) Aragonès, A. C.; Medina, E.; Ferrer-Huerta, M.; Gimeno, N.; Teixidó, M.; Palma, J. L.; Tao, N.; Ugalde, J. M.; Giralt, E.; Díez-Pérez, I.; et al. Measuring the Spin-Polarization Power of a Single Chiral Molecule. Small 2017, 13, No. 1602519.

(48) Eckshtain-Levi, M.; Capua, E.; Refaely-Abramson, S.; Sarkar, S.; Gavrilov, Y.; Mathew, S. P.; Paltiel, Y.; Levy, Y.; Kronik, L.; Naaman, R. Cold denaturation induces inversion of dipole and spin transfer in chiral peptide monolayers. Nat. Commun. 2016, 7, No. 10744.

(49) Miguel, D.; Alvarez de Cienfuegos, L.; Martin-Lasanta, A.; Morcillo, S. P.; Zotti, L. A.; Leary, E.; Burkle, M.; Asai, Y.; Jurado, R.; Cardenas, D. J.; et al. Toward Multiple Conductance Pathways with Heterocycle-Based Oligo(phenyleneethynylene) Derivatives. J. Am. Chem. Soc. 2015, 137, 13818-13826.

(50) Quek, S. Y.; Kamenetska, M.; Steigerwald, M. L.; Choi, H. J.; Louie, S. G.; Hybertsen, M. S.; Neaton, J.; Venkataraman, L. Mechanically controlled binary conductance switching of a singlemolecule junction. Nat. Nanotechnol. 2009, 4, 230-234.

(51) Senanayake, S. D.; Stacchiola, D.; Liu, P.; Mullins, C. B.; Hrbek, J.; Rodriguez, J. A. Interaction of $\mathrm{CO}$ with $\mathrm{OH}$ on $\mathrm{Au}$ (111): HCOO, $\mathrm{CO} 3$, and $\mathrm{HOCO}$ as key intermediates in the water-gas shift reaction. J. Phys. Chem. C 2009, 113, 19536-19544.

(52) Ahlrichs, R.; Br, M.; Hser, M.; Horn, H.; Klmel, C. Electronic structure calculations on workstation computers: The program system turbomole. Chem. Phys. Lett. 1989, 162, 165-169.

(53) Schfer, A.; Horn, H.; Ahlrichs, R. Fully optimized contracted Gaussian basis sets for atoms Li to Kr. J. Chem. Phys. 1992, 97, 25712577.

(54) Perdew, J. P. Density-functional approximation for the correlation energy of the inhomogeneous electron gas. Phys. Rev. B 1986, 33, 8822-8824.

(55) Pauly, F.; Viljas, J. K.; Huniar, U.; Häfner, M.; Wohlthat, S.; Bürkle, M.; Cuevas, J. C.; Schön, G. Cluster-based density-functional approach to quantum transport through molecular and atomic contacts. New J. Phys. 2008, 10, No. 125019.

(56) Zotti, L. A.; Bürkle, M.; Pauly, F.; Lee, W.; Kim, K.; Jeong, W.; Asai, Y.; Reddy, P.; Cuevas, J. C. Heat dissipation and its relation to thermopower in single-molecule junctions. New J. Phys. 2014, 16, No. 015004.

(57) Quek, S. Y.; Venkataraman, L.; Choi, H. J.; Louie, S. G.; Hybertsen, M. S.; Neaton, J. B. Amine-Gold Linked Single-Molecule Circuits: Experiment and Theory. Nano Lett. 2007, 7, 3477-3482.

(58) Grimme, S. Semiempirical GGA-type density functional constructed with a long-range dispersion correction. J. Comput. Chem. 2006, 27, 1787-1799. 